\title{
A NOTE ON COHOMOLOGY ADDITION
}

\author{
P. E. CONNER ${ }^{1}$
}

Professor A. D. Wallace proposed in a Research Problem a question concerning the validity of a cohomology addition theorem [3]. We shall answer his question in the negative. We shall only use the AWS-cohomology groups and the singular homology groups. First a pair of definitions with the coefficient group suppressed. Let $X$ be a Hausdorff space and $h \in H^{n}(X)$ a nonzero cohomology class, then $X$ is a minimal support for $h$ if and only if for every proper closed subset $C \subset X$ the class $h$ lies in the kernel of $i^{*}: H^{n}(X) \rightarrow H^{n}(C)$. Let $A \subset X$ be a closed subset and $h \in H^{n}(A)$ a nonzero class. A closed set $M \subset X$ is an irreducible membrane for $h$ if and only if for every proper closed subset $C \subset M$ the class $h$ lies in the image of $i^{*}: H^{n}(C \cup A) \rightarrow H^{n}(A)$, but is not in the image of $i^{*}: H^{n}(M \cup A) \rightarrow H^{n}(A)$. The two concepts are developed extensively in (1). We now state the problem. Let $X=X_{1} \cup X_{2}$ be a compact Hausdorff space expressed as the union of two closed subsets. If $X_{1} \cap X_{2}$ is a minimal support for some nonzero cohomology class $h \in H^{n}\left(X_{1} \cap X_{2}\right)$ and if $X_{1}$ and $X_{2}$ are both irreducible membranes for $h$, does it then follow that $H^{n+1}(X) \neq 0$ ? We shall show by example that it does not follow. Two points should be noted. First, the question does bear some resemblance to the well-known homology addition theorem, and second if $X=X_{1} \cup X_{2}$ is a finite simplicial complex expressed as the union of two closed subcomplexes then it follows from the remaining hypotheses, with field coefficients, that $H^{n+1}(X) \neq 0$. It is quite possible that the use of field coefficients is unnecessary in this case.

Now for the example. Let $K \subset S^{2}$ be a subcontinuum of the 2sphere which irreducibly separates it, and for which $S^{2}-K=C_{1} \cup C_{2}$ $\cup C_{3}$ is the union of three disjoint open connected sets each of which is homeomorphic to the open 2-cell. Such a continuum is presented in [2, p. 118]. For each component $C_{i}$, the frontier $F\left(C_{i}\right)=K$. Let $X_{i}=C_{i}^{*}=C_{i} \cup K$ for $i=1,2,3$. By the Alexander duality theorem $\tilde{H}^{i}\left(K ; Z_{2}\right) \simeq \tilde{H}_{1-j}\left(S^{2}-K ; Z_{2}\right)$, thus $H^{1}\left(K ; Z_{2}\right) \simeq Z_{2}+Z_{2}$. If $A \subset K$ is a proper closed subset, then $H^{1}\left(A ; Z_{2}\right)=0$, for otherwise $K$ would not irreducibly separate $S^{2}$. We conclude that $K$ is a minimal support for every nontrivial class in $H^{1}\left(K ; Z_{2}\right)$.

The pair $\left(X_{i}, K\right)$ is a relative 2-cell, thus $H^{2}\left(X_{i}, K ; Z_{2}\right) \simeq Z_{2}$, and $H^{i}\left(X_{i}, K ; Z_{2}\right)=0, j \neq 2$. From the exact sequence of pair $\left(X_{i}, K\right)$ we have

Received by the editors May 18, 1961 .

1 The author is an Alfred P. Sloan Fellow. 


$$
0 \rightarrow H^{1}\left(X_{i} ; Z_{2}\right) \rightarrow H^{1}\left(K ; Z_{2}\right) \rightarrow H^{2}\left(X_{i}, K ; Z_{2}\right) \rightarrow 0
$$

from which we see $H^{1}\left(X_{i} ; Z_{2}\right) \simeq Z_{2}$ and $i^{*}: H^{1}\left(X_{i} ; Z_{2}\right) \rightarrow H^{1}\left(K ; Z_{2}\right)$ is a monomorphism. For each $i=1,2,3$ there is a unique nonzero cohomology class $e_{i} \in H^{1}\left(K ; Z_{2}\right)$ which lies in the image of $i^{*}: H^{1}\left(X_{i} ; Z_{2}\right)$ $\rightarrow H^{1}\left(K ; Z_{2}\right)$.

Let $X=X_{1} \cup X_{2}$, then $X$ is a proper subcontinuum of $S^{2}$ which fails to separate, thus $H^{j}\left(X ; Z_{2}\right)=0, j>0$. From the Mayer-Vietoris sequence of $X=X_{1} \cup X_{2}$ we see that $I^{*}: H^{1}\left(X_{1} ; Z_{2}\right)+H^{1}\left(X_{2} ; Z_{2}\right)$ $\simeq H^{1}\left(K ; Z_{2}\right)$. This shows $e_{1} \neq e_{2}$, but even more the three classes $e_{1}, e_{2}, e_{3}$ are distinct and $e_{1}+e_{2}=e_{3}$.

Finally we must show that if $h \in H^{1}\left(K ; Z_{2}\right)$ is not in the image of $i^{*}: H^{1}\left(X_{i} ; Z_{2}\right) \rightarrow H^{1}\left(K ; Z_{2}\right)$ then $X_{i}$ is an irreducible membrane for $h$. In any case there is an irreducible membrane for $h, M \subset X_{i}$ such that $M \supset K[1,1.3,1.4]$. If $M \neq X_{i}$, then $M$ omits an interior point of $X_{i}$. Now the interior of $X_{i}$ is $C_{i}$, an open 2-cell. Around any point in $C_{i}$ we may select a small regular open 2-cell $\sigma \subset C_{i}$ so that $F(\sigma) \cap K$ $=\varnothing$ and $F(\sigma)=S^{1}$. The space obtained from $X_{i}-\sigma$ by collapsing $K$ to a point is a closed 2-cell, thus from excision $H^{j}\left(X_{i}-\sigma, K ; Z_{2}\right)=0$, $j \geqq 0$ and $i^{*}: H^{1}\left(X_{i}-\sigma ; Z_{2}\right) \simeq H^{1}\left(K ; Z_{2}\right)$. We now see that the irreducible membrane $M \subset X_{i}$ cannot omit any interior point of $X_{i}$, thus $M=X_{i}$, an irreducible membrane for $h$.

We note that $X_{1} \cap X_{2}=K$ is a minimal support for $e_{1}+e_{2}$, while $X_{1}, X_{2}$ must both be irreducible membranes for $e_{1}+e_{2}$, however $H^{2}\left(X ; Z_{2}\right)=0$.

\section{REFERENCES}

1. J. W. Keesee, Sets which separate spheres, Proc. Amer. Math. Soc. 5 (1954), 193200.

2. B. v. Kerekjarto, Vorlesungen ueber topologie. I. Flachen topologie, vol. 8, Grundlehrender Mathe. Wissen., J. Springer, 1923, especially pp. 118-120.

3. A. D. Wallace, $A n$ addition theorem for cohomology, Research Problem 11, Bull. Amer. Math. Soc. 61 (1955), 93.

UNIVERSITY OF VIRGINIA 